\title{
Appearance Teasing and Mental Health: Gender Differences and Mediation Effects of Appearance-Based Rejection Sensitivity and Dysmorphic Concerns
}

\author{
Jennifer Schmidt ${ }^{1,2}$ and Alexandra Martin ${ }^{1 *}$ \\ ${ }^{1}$ Clinical Psychology and Psychotherapy, School of Human and Social Sciences, University of Wuppertal, Wuppertal, \\ Germany, ${ }^{2}$ Department of Psychology, HSD Hochschule Döpfer University of Applied Sciences, Cologne, Germany
}

OPEN ACCESS

Edited by:

Silja Vocks,

Osnabrück University, Germany

Reviewed by:

Andrea Wyssen,

Université de Fribourg, Switzerland Erin Elizabeth Reilly, University of California, San Diego,

United States

*Correspondence: Alexandra Martin martin@uni-wuppertal.de

Specialty section: This article was submitted to

Eating Behavior,

a section of the journal

Frontiers in Psychology

Received: 13 December 2018 Accepted: 01 March 2019

Published: 20 March 2019

Citation:

Schmidt J and Martin A (2019) Appearance Teasing and Mental

Health: Gender Differences and Mediation Effects

of Appearance-Based Rejection Sensitivity and Dysmorphic Concerns.

Front. Psychol. 10:579. doi: 10.3389/fpsyg.2019.00579
Appearance teasing is a common phenomenon in social interactions, especially in adolescence. Several studies have shown its negative impact on mental health as well as on body image. While these findings prove stable in various contexts, less evidence is available for possible gender differences in these relationships. In particular, the role of two important body image variables - appearance-based rejection sensitivity (ARS) and dysmorphic concerns - and their contribution to mental health impairments has not been assessed in gender-specific process models. In a cross-sectional survey-study ( $N=501 ; 407$ f, 94 m), we retrospectively assessed early appearance teasing experiences, as well as current ARS, dysmorphic concerns, depression, anxiety, and self-esteem. We analyzed gender differences in these variables and their interrelations. We then examined the mediating role of ARS and dysmorphic concerns in explaining mental health variables in adulthood due to early appearance teasing in gender-specific serial-mediation models. The results show high ratios of early teasing experiences, but no significant gender difference regarding the frequency of early appearance teasing. While teasing experiences were significantly related to body image variables in adulthood in both genders ( $r s>0.32$; ps < 0.010), we observed significant relations with mental health outcomes in women ( $r s>0.30$; ps $<0.001$ ) but not in men ( $r s<0.20$; ps $>0.250$ ). Serial mediation models show that ARS and dysmorphic concerns mediate the effects of appearance teasing on mental health in all outcomes in women $\left(\Delta R^{2}>0.17\right)$, but not in men $\left(\Delta R^{2}<0.03\right)$. Findings remained stable when controlling for Body-Mass-Index, age, and relationship-status. The findings show similar frequencies of appearance teasing and associated negative effects on body image in men and women. Specifically, in women, the effects of teasing on mental health were stronger and mediated by ARS and dysmorphic concerns. Overall, the results point to 
the relevance of ARS for etiological models of body image disorders and female mental health. However, men did not show the same relationships of teasing and mental health. Differential resilience factors regarding the negative effects of early appearance teasing could be an important target for future research.

Keywords: appearance teasing, mental health, body image, gender differences, body dysmorphic disorder, appearance-based rejection sensitivity

\section{INTRODUCTION}

Appearance teasing (i.e., negative social feedback on one's physical characteristics; Cash, 1995) is a common phenomenon, to which a great number of individuals is exposed during childhood and adolescence. It often occurs in the form of verbal harassments and provocations, ranging from name-calling (e.g., "fatso," "pizza face," or "wimp") to hostile remarks. In this context, teasing needs to be distinguished from victimization and bullying behaviors with a more physical and violent nature, such as sexual harassment, severe threats, or physical violence (e.g., Kochenderfer and Ladd, 1996; Keltner et al., 2001).

Studies on the prevalence, types, and effects of appearance-related teasing experiences show that approximately three-quarters of college students reported such teasing experiences (Cash, 1995; Hayden-Wade et al., 2005; Almenara and Ježek, 2015). Regarding the target of teasing, experiences often relate to the individual's body weight (e.g., Cash, 1995; Hayden-Wade et al., 2005). However, appearance teasing can also relate to facial features (e.g., the nose), body height, chest, hair, or extremities (Cash, 1995). Sources of teasing experiences are peers (up to 60\%), but also siblings (up to 36\%) and parents (up to 19\%) (Cash, 1995; Keery et al., 2005).

There is inconsistent evidence with regard to gender-specific occurrence of appearance teasing. For example, some studies report higher teasing frequencies in girls compared to boys (Farrow and Fox, 2011; Almenara and Ježek, 2015). Other studies report higher rates of verbal bullying and appearance pressure reported by boys compared to girls (Jones and Crawford, 2006; Stubbs-Richardson et al., 2018) or no gender differences at all (Schwartz et al., 1999; Phares et al., 2004). These contradictory results might emerge from a great heterogeneity in studied samples with respect to age, cultural background, and different conceptualizations of teasing (e.g., Liang et al., 2011).

While the numbers suggest that appearance teasing is a common and widespread phenomenon, it would be a mistake to discount its meaning and severity. Many studies with focus on the examination of appearance teasing showed negative effects on several mental health outcomes. These encompass impairments in self-esteem, increased levels of depression (Jackson et al., 2000; Faith et al., 2008; Greenleaf et al., 2014; Feragen and Stock, 2016), and even suicidal ideation and attempts (Eisenberg et al., 2003; Ford et al., 2017). In adolescents, teasing experiences are associated with social avoidance, fear of negative evaluation, and loneliness (Storch et al., 2003). McCabe et al. (2010) reported associations of retrospective accounts of teasing during childhood and anxiety disorders in adults, especially social anxiety. In addition, disordered eating, psychosomatic problems, and physical health show relations with appearance teasing experiences (Keery et al., 2005; Gini and Pozzoli, 2009; Thompson et al., 2018). Overall, the negative impact of general types of victimization on psychosocial adjustments (operationalized as depression, anxiety, self-esteem) is well established (Hawker and Boulton, 2000). While appearance teasing thus poses a high risk of negative outcomes in individuals reporting teasing, evidence on possible gender differences is - again - less consistent. Some researchers reported stronger and broader negative outcomes of teasing in girls (Jones et al., 2005; Gruber and Fineran, 2008; CarboneLopez et al., 2010; Feragen and Stock, 2016), while others found gender-invariant effects (Eisenberg et al., 2003).

With regard to the more proximal psychological consequences, adolescent appearance teasing experiences have significant effects on body image concerns (Menzel et al., 2010). Two important constructs that have been highlighted in this regard are the cognitive processes of dysmorphic concerns and the personality disposition of appearance-based rejection sensitivity (ARS).

Dysmorphic concerns are a core symptom of body dysmorphic disorder (BDD) and describe excessive and time consuming cognitive preoccupation with one's appearance (Phillips, 2005; American Psychiatric Association, 2013). Empirical findings from cross-sectional and longitudinal studies show that early appearance teasing experiences are associated with more dysmorphic concerns (Lavell et al., 2014; Webb et al., 2015; Weingarden and Renshaw, 2016; ZimmerGembeck et al., 2018). In addition, patients diagnosed with BDD retrospectively report higher frequencies of appearance teasing than healthy controls (Buhlmann et al., 2007; Buhlmann et al., 2011; Weingarden and Renshaw, 2016; Weingarden et al., 2017). Correspondingly, etiological models of BDD consider appearance teasing as a risk factor for the development of this mental disorder (Veale, 2004; Neziroglu et al., 2008). BDD symptoms in turn relate to poor mental health, with increased degrees of depression, anxiety, and low self-esteem (e.g., Biby, 1998; Bohne et al., 2002).

As another proximal factor, appearance teasing can influence ARS - the anticipation or fear of social rejection because of one's appearance. ARS describes the personality disposition to readily expect and be concerned about interpersonal rejection because of one's physical attractiveness (Park, 2007). Appearance teasing experiences are related to higher levels of ARS (Lavell et al., 2014; Webb et al., 2015, 2017). In itself, ARS is related to depression, anxiety, and low self-esteem (Park et al., 2010; Bowker et al., 2013; Schmidt and Martin, 2017). Further, as a personality disposition, ARS predicts excessive body image 
concerns (Park et al., 2009; Calogero et al., 2010), is elevated in individuals with BDD (Kelly et al., 2014; Schmidt and Martin, 2017), and explains unique variance in BDD symptom severity in comparison to general rejection sensitivity (Kelly et al., 2014). Recent studies provided increasing evidence for the particular relevance of ARS as a mediator between the effects of appearance teasing and dysmorphic concerns (Lavell et al., 2014; Webb et al., 2015, 2017; Densham et al., 2017).

Given the significant relationship of appearance teasing with negative mental health outcomes as well as their strong effects on body image, a serial indirect mediation effect (i.e., a mediation via two or more mediators that are causally and closely associated due to theoretical conceptions and/or empirical findings) of current ARS and dysmorphic concerns might be present. In the relationship of these two body image variables, the personality disposition of ARS should precede the cognitive processes of dysmorphic concerns, as previous studies have established (Lavell et al., 2014; Webb et al., 2015; Densham et al., 2017). Thus, we determined the order of the assumed mediation as: retrospective appearance teasing $\rightarrow$ current ARS $\rightarrow$ current dysmorphic concerns $\rightarrow$ current mental health.

With regard to gender differences in this pathway model, evidence is fragmented and inconclusive. Menzel et al. (2010) did not find gender differences regarding the associations of appearance teasing with body image in their meta-analysis. In addition, Webb et al. (2015) did not identify moderating influences of gender on the effects of teasing on BDD symptoms. However, some evidence suggests gender differences in specific parts of the assumed mediation model, especially regarding the influence of body image variables on mental health. Vogt Yuan (2010) has shown that body perceptions were associated with changes in psychological well-being in a group of female, but not of male adolescents. Other researchers found that self-esteem of young males was not significantly affected by body image and that boys seem to be less sensitive to teasing in general (Bolognini et al., 1996; Gleason et al., 2000; Furnham et al., 2002; Baudson et al., 2016). With regard to depression as another mental health marker, weaker associations with body image in boys compared to girls have been reported (Marcotte et al., 2002; Hyde et al., 2008). These gender differences might be caused by differential importance of appearance for the overall self-evaluation of male and female individuals, for example, due to media-conveyed beauty ideals (e.g., Lopez-Guimera et al., 2010) and the gender-specific importance of appearance for self-esteem (e.g., Gentile et al., 2009). However, many studies examining appearance teasing effects on body image focused on female samples (Menzel et al., 2010) and even in mixed samples, researchers mostly did not specifically address gender differences (e.g., McCabe et al., 2010).

The aim of the present study was therefore twofold: Firstly, we aimed at examining general gender differences in retrospective appearance teasing experiences in childhood and adolescence, current body image, and mental health because of the ambiguous existing results. In line with previous studies on mental health and teasing (e.g., Hawker and Boulton, 2000; Eisenberg et al., 2003), we operationalized mental health via assessments of depression, anxiety, and self-esteem. In addition, we intended to examine the bivariate relationships between these variables and possible gender differences in the strength and significance of the associations.

Secondly, we aimed at analyzing the postulated mediation effect, that is, whether ARS and dysmorphic concerns mediate the relationship of retrospective appearance teasing in childhood and adolescence and current mental health problems. Based on the results of earlier research on mediation effects in single parts of the hypothesized relationships (Lavell et al., 2014; Webb et al., 2015; Densham et al., 2017), we assumed a serial mediation model (appearance teasing $\rightarrow$ ARS $\rightarrow$ dysmorphic concerns $\rightarrow$ mental health). We performed separate gender-specific model analyses to identify whether indirect effects differ between male and female subsamples due to possible gender differences in the relation of body image variables and mental health (e.g., Gleason et al., 2000; Furnham et al., 2002; Hyde et al., 2008; Vogt Yuan, 2010). Altogether, the small number of existing studies does not allow to state definite hypotheses regarding size and significance of gender-specific indirect effects. Therefore, we conduct the gender-separated mediation analyses in an exploratory approach.

To assure statistical robustness of the findings, we reanalyze the models while controlling for possible confounding variables that have shown strong associations with teasing and mental health in earlier research: (1) Body-Mass-Index (BMI; e.g., Hayden-Wade et al., 2005; McLaren et al., 2008), (2) age (e.g., Kessler et al., 2010), and (3) relationship status (e.g., Willitts et al., 2004).

\section{MATERIALS AND METHODS}

\section{Study Design}

The cross-sectional study was conducted online via survey in a German-speaking community sample, using the platform SoSci Survey (Leiner, 2018). Data collection took place from April 4th to May 18th 2018. The study was conducted in line with the Helsinki Declaration and was approved by the research ethics committee of the University of Wuppertal. The survey was announced as a study on appearance, body image, dermatological conditions, and mental health. Aside from the research questions that are addressed in this report, the study also served for the psychometric evaluation of a questionnaire assessing skin picking, which will be the subject of another manuscript planned. The present report is the first presenting results from the project.

\section{Participants}

The target sample was a German-speaking community sample with a broad range of participants regarding age, professions, and health status. Therefore, the only inclusion criteria for study participation were legal age ( $\geq 18$ years) and sufficient knowledge of the German language. All participants provided informed consent on the study participation.

We conducted the recruitment via the local universitynewsletter, other student newsletters, flyers, and social media-websites (Facebook, Xing, What's App-groups, Kleiderkreisel.de), and via the website of a German magazine on psychological topics (Psychologie heute). Psychology students 
at the University of Wuppertal could receive course credit for participation. All other respondents could participate in a raffle of ten 10 EUR gift-cards.

Power calculations with G*Power 3.0 (Faul et al., 2007) showed that the detection of between-group gender differences with small to medium effect sizes $(d=0.35$; see Hawker and Boulton, 2000) would require a minimum sample size of $N=204$, given a significance level of $\alpha=0.05$ and statistical power of $1-\beta=0.80$. For regression-based mediation analyses, a priori power calculation resulted in a sample size of $n=77$ (per group) necessary to detect medium effects $\left(f^{2}=0.15\right)$ at the same significance and power levels in linear regression models with three predictors. Allowing for additional dropout and exclusion-margins, we aimed at recruiting an overall sample of $N>300$.

\section{Assessment Instruments Sociodemographic Data}

To assess sociodemographic characteristics of the sample, the questionnaire included items on the participant's gender (male/female/other), current age (in years), current body weight (in $\mathrm{kg}$ ) and height (in $\mathrm{cm}$ ) for the calculation of the BMI (in $\left.\mathrm{kg} / \mathrm{m}^{2}\right)$. Further questions assessed the current relationship status in a binary format (yes / no), the level of school education, and the current employment status.

\section{Appearance Teasing Experiences}

To assess appearance teasing, an initial question asked whether the participants had ever experienced any severe teasing or bullying/mobbing throughout their lives (yes/no).

Appearance-related teasing was then assessed with a modified version of the weight-teasing subscale of the Perceptions of Teasing Scale (POTS, Thompson et al., 1995; German version: Losekam et al., 2017). The POTS is a commonly used measure to retrospectively assess teasing experiences during childhood and adolescence in adult samples (for an overview, see Menzel et al., 2010). The adapted subscale assesses the frequency and effects of appearance-related teasing experiences in childhood and adolescence. It consists of six items that ask about the teasing experiences while growing up (age 5-16). For each item, respondents can use 5-point Likert scales to indicate (a) the frequency of described situations $(1=$ Never, 3 = Sometimes, 5 = Very often) and (b) the effects ("How upset were you?" $1=$ Not upset, $3=$ Somewhat upset, $5=$ Very upset $)$. Separate mean scores on teasing frequency (WT-F) and teasing effects (WT-E) indicate more frequent teasing-experiences and stronger effects of these experiences (Thompson et al., 1995). The POTS has good psychometric properties, with a good internal consistency (Cronbach's $\alpha>0.87$ ), high test-retest-reliabilities (WT-F: $r_{t t}=0.90$; WT-E: $r_{t t}=0.85$ ), and evidence for its concurrent validity in adult and preadolescent samples (Thompson et al., 1995; Jensen and Steele, 2010).

For the purpose of the present study, whenever the original items referred to weight as the cause of the teasing, we replaced the respective words with more general terms that address the overall appearance (e.g., "People made fun of you because you were heavy" was changed to "People made fun of you because of your appearance"). We only used the teasing frequency (POTS-TF) subscale for analyses. The internal consistency of the POTS-TF was good in the present sample $(\alpha=0.89)$.

\section{Appearance-Related Mediators \\ Dysmorphic concerns}

We assessed dysmorphic concerns with the Dysmorphic Concerns Questionnaire (DCQ; German version: Stangier et al., 2003). The DCQ is a frequently used screening instrument to assess cognitive appearance concerns and symptoms of BDD via self-report. The scale consists of seven items to be answered on 4-point Likert scales $(0=$ not at all; $3=$ much more than other people). Here, higher values of the sum score indicate more dysmorphic concerns. The one-factorial instrument has a good reliability and validity (Schieber et al., 2018), and showed a good internal consistency in the present sample $(\alpha=0.85)$.

\section{Appearance-based rejection sensitivity}

The personality disposition of ARS was assessed with the Appearance-Based Rejection Sensitivity Scale (ARS-D; Park, 2007; German version: Schmidt and Martin, 2017). The shortform of the ARS-D consists of twelve items that describe brief scenarios related to one's appearance in various types of social contexts, for example, "You are leaving your house to go on a first date when you notice a blemish on your face." For each scenario, respondents can use 6-point rating scales to indicate (a) their worries about rejections due to the appearance in the scenario (affective component; $1=$ very unconcerned; $6=$ very concerned) and (b) the estimated likelihood of such rejection experiences (cognitive component; $1=$ very unlikely; 6 = very likely). The scores of the affective and the cognitive component of each scenario are multiplied and averaged, leading to a mean score with a range of 1 to 36 . Higher values indicate stronger ARS. Psychometric properties of the ARS-D are good with high internal consistency ( $\alpha=0.90$; Schmidt and Martin, 2017; $\alpha=0.92$ in the present sample) and convergent and discriminant validity (Park, 2007; Schmidt and Martin, 2017).

\section{Mental Health Outcomes}

Three different constructs served as multifactorial indicators of the current state of respondents' mental health: depression, anxiety, and self-esteem. The respective questionnaires were based on self-report.

\section{Depression}

We applied the German version of the Patient Health Questionnaire-9 Depression module (PHQ-9; Gräfe et al., 2004) to assess current depressive symptoms. The PHQ-9 contains nine items to rate current symptoms of major depression in line with the respective diagnostic criteria with regard to the previous 2 weeks. The frequency of symptoms is answered on a 4-point rating scale $(0=$ not at all; $3=$ nearly every day $)$, and the resulting sum score indicates the severity of depressive symptoms. The PHQ-9 is a widely used and valid screener to assess depressive symptoms (Martin et al., 2006) and shows a high internal consistency ( $\alpha=0.88$, Gräfe et al., 2004; $\alpha=0.89$ in the present sample). 


\section{Anxiety}

For the assessment of current symptoms of anxiety, we applied the anxiety module of the Patient Health Questionnaire; the Generalized Anxiety Disorder Scale (GAD-7; Löwe et al., 2008). The GAD-7 assesses typical symptoms of anxiety (e.g., worries, tension, difficulties to relax etc.) on seven items regarding the last 2 weeks. Answers are provided on 4-point rating scales $(0=n o t$ at all; $3=$ nearly every day). The overall sum score indicates the severity of anxiety symptoms. The brief economic instrument has shown factorial and construct validity, as well as a very good to excellent reliability ( $\alpha=0.88$, Löwe et al., $2008 ; \alpha=0.90$ in the present sample).

\section{Self-esteem}

Self-esteem was assessed with the German version of the Rosenberg Self Esteem Scale (RSES; von Collani and Herzberg, 2003). The ten-item questionnaire assesses global self-esteem as a stable trait, using 4 -point rating scales $(0=$ strongly disagree; $3=$ strongly agree). After recoding inverted items, the score is summed up with higher values indicating higher self-esteem. The psychometric properties of the instrument are good and correlations with other mental health outcomes are significant (von Collani and Herzberg, 2003; Sinclair et al., 2010). Internal consistency is reported as very good ( $\alpha=0.85$, von Collani and Herzberg, 2003), with even higher reliability in the present sample $(\alpha=0.91)$.

\section{Procedure}

Volunteers were able to participate in the study via clicking on a respective hyperlink that was distributed in the recruitment process. They first had to read the study information and provide informed consent. The questionnaire then started with an assessment of the sociodemographic characteristics. This assessment was followed by questions about dermatological problems and skin picking behaviors, to answer another research question. Then, the ARS-D, POTS, RSES, DCQ, GAD-7, and PHQ-9 were administered in the stated order. Intermittently, we included motivational messages to retain the participants' attention and involvement. A progress bar provided an overview on the percentage of completed questions.

Overall, it was possible to skip items if the respondents were not willing to answer the questions. In this case, a pop up asked whether the items had been left out on purpose. This procedure was chosen to avoid dropout because of reluctance to answer certain sensitive questions. At all time, a contact e-mail address was displayed in case of any further questions or the need to contact the researchers. Overall, it took approx. 20-30 min to fill in the complete questionnaire.

\section{Data Processing}

A total of $N=838$ respondents first opened the survey website, whereof $N=532$ completed the survey (i.e., response at the final webpage; dropout rate: $36.5 \%)$. We deleted the data of participants who indicated an age $<18$ or no age at all $(n=2)$, in line with the inclusion criteria of the study. We screened the provided response time measures and normed data quality measures provided by the online software (DEG_TIME; Leiner, 2018) for suspicious working speed (TIME_SUM criterion; one third of the average time in test runs; $<9 \mathrm{~min}$ ) and low data quality (DEG_TIME criterion $<100$ ) when filling in the questionnaire. We excluded $n=25$ datasets due to insufficient data quality. Because of the research question of this study and the small number of individuals who indicated another gender than male or female $(n=3)$, we excluded these cases from the analyses. Another participant was excluded because she did not indicate a weight or height for the calculation of the BMI as a control variable.

Altogether, the amount of missing values in the overall survey was $<0.15 \%$. There were no datasets with more than $50 \%$ of missing values in any of the applied assessment instruments. For other single missing values, we conducted the Missing Completely at Random-Test (MCAR; Little, 1988). The test indicated that there were no systematic patterns in the missing values. We then used a multiple imputation technique $(m=20)$ to replace missing values. The resulting estimates for all 20 imputed datasets were manually compared to the means in the original dataset for all 123 variables with missing values. We aimed at selecting the imputed dataset with the least deviation from the means in the original dataset. Imputation 11 showed deviations from the original dataset in only 17 variables. In 14 of these variables, the deviation from the original mean was 0.01 points and in the remaining three variables, the deviation was 0.02 points, indicating very small deviations. The data were screened regarding extreme outliers in the relevant variables, using box plot analyses. However, no participant had to be excluded for this reason. The final analysis sample consisted of $N=501$ datasets, with $n=407$ female and $n=94$ male respondents.

\section{Statistical Analyses}

Sample characteristics regarding the sociodemographic data and former teasing experiences were calculated as frequencies or means of the female and male subgroups and compared between genders by means of $t$-test and $\chi^{2}$-tests. We also performed gender comparisons for the means of all relevant variables in analyses (POTS-TF, DCQ, ARS-D, PHQ-9, GAD-7, RSES) with $t$-tests. However, because Kolmogorov-Smirnov tests and Levene's tests showed that the assumptions of normality and homoscedasticity were violated for the majority of variables, we backed up the results of those variables with non-parametric Mann-Whitney- $U$-Tests.

We then calculated two-sided correlations to analyze the general relationship between teasing (POTS-TF), mediator variables (DCQ, ARS-D), and mental health outcomes (PHQ-9, GAD-7, RSES) separately for the male and female subgroups, using Spearman correlations with Bonferroni-corrections to adjust for multiple comparisons. Fisher's $Z$-tests served to analyze potential gender differences in the size of the resulting correlation coefficients of the POTS and the other outcomes.

Mediation analyses were conducted with PROCESS for SPSS (Hayes, 2013), using a serial mediation model (model 6) with retrospective teasing frequency (POTS-TF) as predictor, two serial mediators (ARS-D $\rightarrow$ DCQ), and mental health variables as outcomes (PHQ-9, GAD-7, RSES). The conceptual 


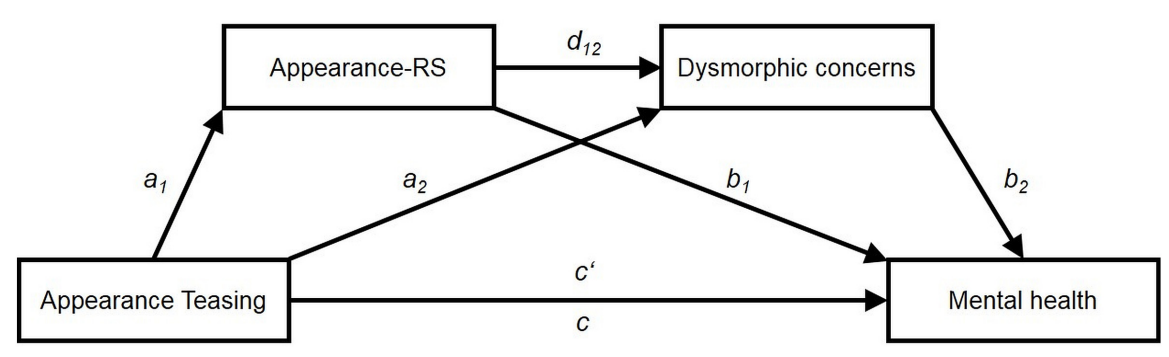

FIGURE 1 | Conceptual mediation model. Appearance-RS, appearance-based rejection sensitivity.

model (see Figure 1) is based on three linear regression analyses. The first regression analysis tests the effects of appearance teasing frequency (POTS-TF) on ARS (ARS-D values; path $\mathrm{a}_{1}$ in Figure 1). The second regression model tests the combined predictive effects of appearance teasing and ARS on dysmorphic concerns (DCQ values; paths $\mathrm{a}_{2}$ and $\mathrm{d}_{12}$ in Figure 1). The third regression predicts the mental health outcome (PHQ-9, GAD-7, RSES) by the independent variable (POTS-TF) and the two mediators (paths $b_{1}, b_{2}$, and $c^{\prime}$ in Figure 1). Here, path $c^{\prime}$ in Figure $\mathbf{1}$ depicts the direct effect of appearance teasing on the mental health outcomes controlled for the effects of the two mediators. In contrast, path $c$ in Figure $\mathbf{1}$ indicates the total effect of appearance teasing on mental health outcomes without considering the mediators.

For each mental health outcome, we calculated separate models based on the male and female subgroups. The indirect effects were evaluated using $n=5000$ bootstrap samples. Further, to rule out effects of heteroscedasticity, the analyses used the HC3 estimator for heteroscedasticity-consistent standard errors. The significance of the indirect effects was tested by using $95 \%$ confidence intervals.

To test for the robustness of the mediation analyses and to rule out possible and previously shown influences of age, $\mathrm{BMI}$, and the current relationship-status on mediators and mental health outcomes (e.g., Willitts et al., 2004; McLaren et al., 2008; Kessler et al., 2010), all mediation analyses were subsequently repeated, controlling for the above mentioned variables as covariates.

Overall, the significance level was defined as $p<0.05$. To classify the effect size of possible gender differences, Hedge's $g$ for different group sizes was calculated. Conventions for $g$ are that values of $g \geq 0.20$ indicate small effects, values of $g \geq 0.50$ indicate medium, and values of $g \geq 0.80$ indicate large effects (Cohen, 1988).

\section{RESULTS}

\section{Sample Characteristics}

The final analysis sample $(N=501 ; 407$ female, 94 male $)$ had an overall age range from 18 to 74 years with a mean age of 31.2 years $(S D=11.7)$ and a mean BMI in the normal weight range $\left(M=23.9 \mathrm{~kg} / \mathrm{m}^{2}, S D=5.5 \mathrm{~kg} / \mathrm{m}^{2}\right)$.
The majority of the sample was in a relationship with a partner (65.7\%) and currently working (68.7\%). Overall, $42.7 \%$ of the sample were students, $54.7 \%$ were employed, $7.8 \%$ were self-employed, $4.4 \%$ were jobless or incapable of working, $1.6 \%$ were retired (multiple answers were possible). The education level of the sample was generally high: $78.4 \%$ of the participants had a university entrance diploma, $12 \%$ had an advanced college entrance qualification, $8.2 \%$ had a secondary school certificate and $1.4 \%$ had other qualifications. Almost half of the sample (44.5\%) reported that they had experienced severe teasing, bullying, or mobbing at least once in their lives.

\section{Gender Group Differences}

A detailed overview on gender differences is provided in Tables 1, 2. The male subgroup was slightly older and had a higher BMI than the female subgroup $(p s<0.031)$. However, the distribution of relationship status, school education, employment, and the ratio of any teasing/bullying experiences did not differ between genders ( $p s>0.547$ ).

The $t$-test indicated that the frequency of early appearance-related teasing experiences (POTS-TF) in childhood and adolescence was higher in women compared to men. However, the effect size of the group difference was small $(g=0.25)$, and the group difference was no longer significant when applying non-parametric tests on group differences $(Z=-1.84, p=0.066)$. For the other appearance-related variables, dysmorphic concerns and ARS were higher in women compared to men with medium effect sizes (DCQ: $g=0.54$; ARS-D: $g=0.57$ ).

With regard to mental health variables, women showed significantly higher degrees of depression $(g=0.42)$, anxiety $(g=0.43)$, and lower self-esteem $(g=0.29)$ than men, with small to medium effect sizes. Non-parametric analyses confirmed the significant gender differences in these variables (all $p s<0.013$ ).

\section{Correlations}

As displayed in Table 3, the non-parametric correlation analyses in the female subgroup showed medium-sized relationships of appearance teasing frequency (POTS-TF) with dysmorphic concerns and with ARS ( $p s<0.001)$. Further, the relationships of the POTS-TF and the three mental health variables were highly significant $(p s<0.001)$. Additionally, the appearance-related mediator variables and 
TABLE 1 | Sample characteristics of the female and male subgroup in sociodemographic data.

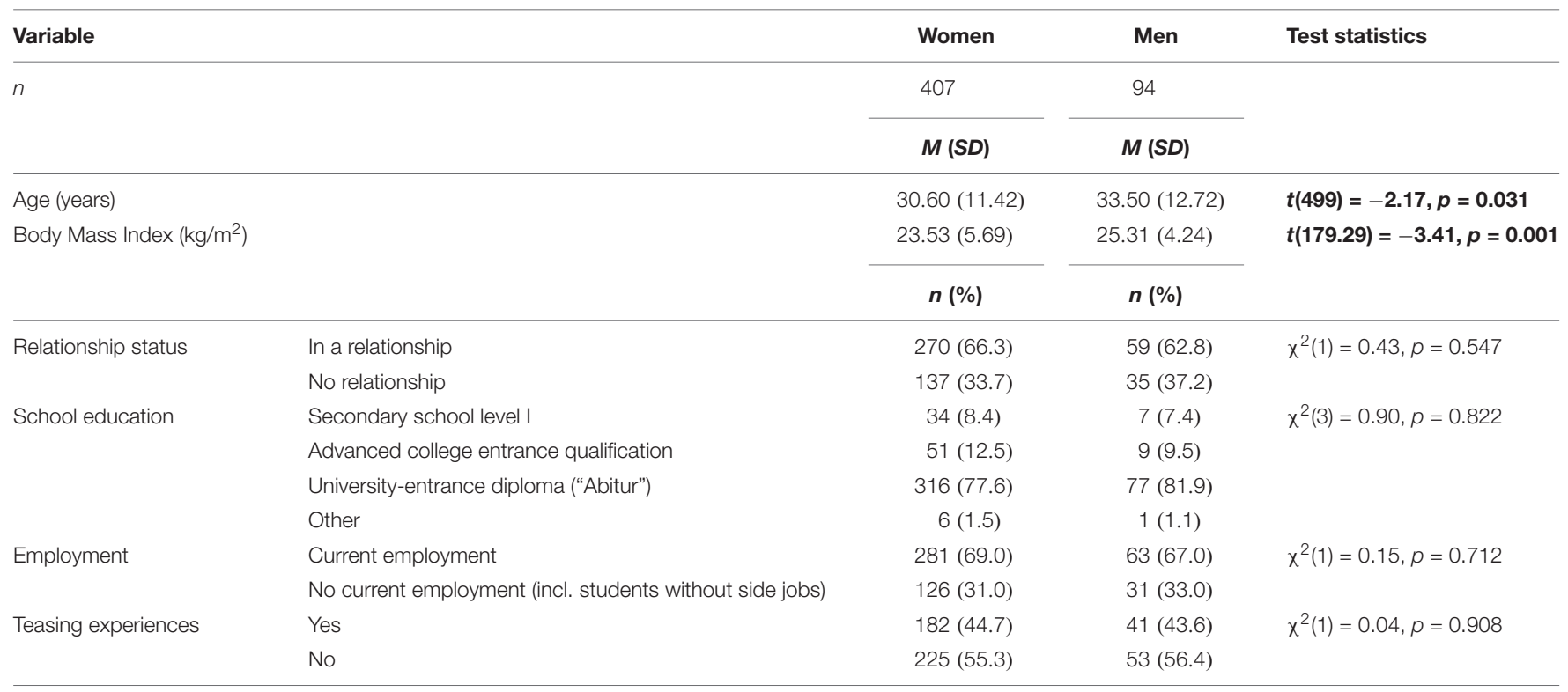

Boldface entries indicate significant gender group differences, two-sided $p<0.05$. In case of unequal variances in Levene's test, degrees of freedom were adjusted.

TABLE 2 | Gender differences in teasing frequency, body image, and mental health (mean, standard deviations, and test statistics).

\begin{tabular}{|c|c|c|c|c|}
\hline Variable & Women & Men & Levene's test & Test statistics \\
\hline$n$ & 407 & 94 & & \\
\hline \multirow[t]{2}{*}{ Appearance-teasing frequency (POTS) } & 2.09 & 1.86 & $F=8.02$ & $t(166.63)=2.47, p=0.015^{a}$ \\
\hline & $(0.95)$ & $(0.76)$ & $p=0.005$ & \\
\hline \multirow[t]{2}{*}{ Appearance concerns (DCQ) } & 8.23 & 5.77 & $F=10.85$ & $t(163.66)=5.27, p<0.001$ \\
\hline & $(4.75)$ & $(3.89)$ & $p=0.001$ & \\
\hline \multirow[t]{2}{*}{ Appearance-based rejection sensitivity (ARS-D) } & 12.63 & 8.46 & $F=19.29$ & $t(194.03)=6.22, p<0.001$ \\
\hline & $(7.72)$ & $(5.34)$ & $p<0.001$ & \\
\hline \multirow[t]{2}{*}{ Depression (PHQ-9) } & 7.77 & 5.44 & $F=5.18$ & $t(160.07)=4.08, p<0.001$ \\
\hline & $(5.73)$ & $(4.81)$ & $p=0.023$ & \\
\hline \multirow[t]{2}{*}{ Anxiety (GAD-7) } & 7.33 & 5.20 & $F=5.91$ & $t(157.29)=4.29, p<0.001$ \\
\hline & $(5.03)$ & $(4.30)$ & $p<0.001$ & \\
\hline \multirow[t]{2}{*}{ Self-Esteem (RSES) } & 21.29 & 23.12 & $F=4.11$ & $t(156.71)=-2.78, p=0.006$ \\
\hline & $(6.49)$ & $(5.58)$ & $p=0.043$ & \\
\hline
\end{tabular}

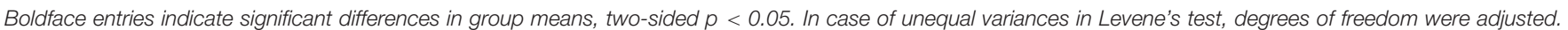

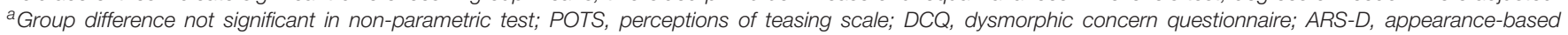

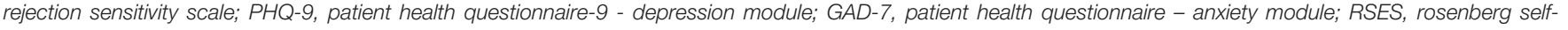
esteem scale.

the mental health variables showed high interrelationships ( $r s>0.52$, ps $<0.001)$.

In the male subsample, appearance teasing frequency also showed medium relationships with dysmorphic concerns and ARS $(p s<0.010)$. However, the correlations of POTS-TF with mental health variables were small $(r s \leq 0.20)$ and not significant after Bonferroni-corrections ( $p s>0.250$ ).

The direct comparisons of the gender-specific correlation coefficients via Fisher-Z-tests showed that the relationship of teasing and depression was significantly higher in women than in men $(p=0.025)$, and there was a trend towards significance for anxiety $(p=0.055)$. The size of the correlation coefficients for teasing frequency and body image variables or self-esteem did not differ significantly between males and females.

\section{Mediation Analyses}

The mediation models for the female and male subsamples are displayed in Figure 2, and model indices are depicted in Table 4. For the female subsample, mediation analyses showed that early appearance teasing increased current ARS $\left(a_{1}=3.20\right.$, $p<0.001)$ and that both, early teasing $\left(a_{2}=1.42, p<0.001\right)$ and ARS $\left(d_{12}=0.24, p<0.001\right)$ predicted dysmorphic concerns. In addition, dysmorphic concerns and ARS were significant mediators for the effects of early appearance teasing frequency on all three mental health variables. 
TABLE 3 | Gender-specific correlations between teasing, body image, and mental health variables and gender differences in correlations.

\begin{tabular}{|c|c|c|c|c|c|c|c|}
\hline Variable & 1 & 2 & 3 & 4 & 5 & 6 & $\begin{array}{c}\text { Gender } \\
\text { differences } \\
\text { (POTS - Variable) }\end{array}$ \\
\hline (2) Dysmorphic concerns (DCQ) & $0.45^{* *}$ & - & $0.37^{* *}$ & 0.24 & 0.26 & -0.24 & $Z=0.42, p=0.336$ \\
\hline (3) Appearance-based rejection sensitivity (ARS-D) & $0.38^{* *}$ & $0.52^{* *}$ & - & 0.06 & 0.07 & -0.26 & $Z=0.59, p=0.278$ \\
\hline (4) Depression (PHQ-9) & $0.38^{* *}$ & $0.51^{* *}$ & $0.43^{* *}$ & - & $0.72^{* *}$ & $-0.48^{* *}$ & $Z=1.97, p=0.025^{*}$ \\
\hline
\end{tabular}

Correlation coefficients are based on Spearman's correlation, two-sided; Values below the diagonal base on the female subgroup, values above the diagonal refer to the male subgroup. POTS, perceptions of teasing scale; DCQ, dysmorphic concern questionnaire; ARS-D, appearance-based rejection sensitivity scale; PHQ-9, patient health questionnaire-9 - depression module; GAD-7, patient health questionnaire - anxiety module; RSES, modified Rosenberg self-esteem scale. Indications of significance base on Bonferroni-corrected p-values; Gender differences regarding the correlations of POTS and other variables were assessed via Fisher's-Z-test; $* p<0.05 ; * *<0.01$.

\section{Women $(n=407)$}
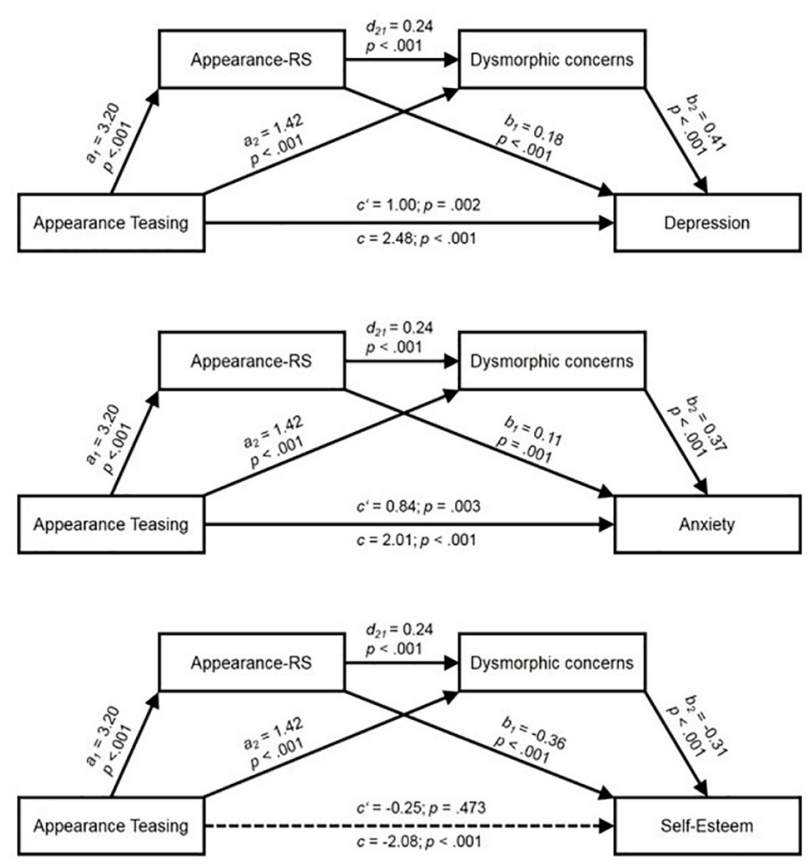

\section{Men $(n=94)$}
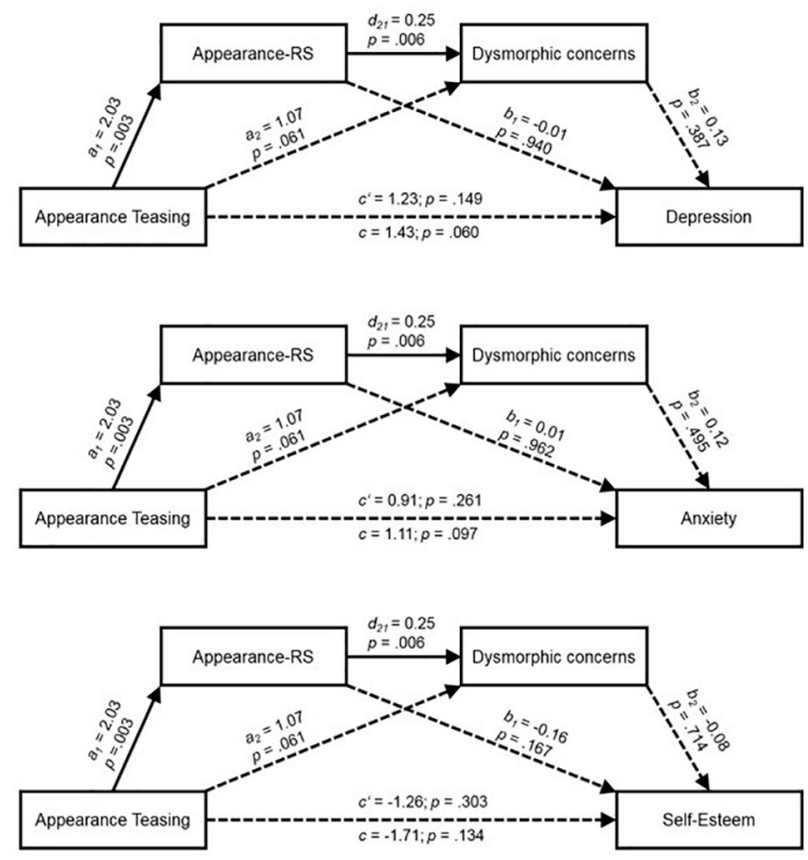

FIGURE 2 | Gender-specific serial mediation models. Appearance-RS, appearace-based rejection sensitivity.

TABLE 4 | Comparison of gender-specific model indices for the total effect and serial mediation models in the female and male subgroups.

\begin{tabular}{|c|c|c|c|c|c|c|c|c|}
\hline \multirow[t]{2}{*}{ Model characteristics } & \multicolumn{4}{|c|}{ Female $(n=407)$} & \multicolumn{4}{|c|}{ Male $(n=94)$} \\
\hline & $\boldsymbol{F}$ & $d f$ & $p$ & $R^{2}$ & $\boldsymbol{F}$ & $d f$ & $p$ & $R^{2}$ \\
\hline A-Teasing $\rightarrow$ Depression & 57.9 & 1,405 & $<0.001$ & 0.17 & 3.6 & 1,92 & 0.060 & 0.05 \\
\hline A-Teasing $\rightarrow$ ARS $\rightarrow$ DC $\rightarrow$ Depression & 67.1 & 3,403 & $<0.001$ & 0.37 & 1.5 & 3,90 & 0.223 & 0.06 \\
\hline A-Teasing $\rightarrow$ Anxiety & 48.0 & 1,405 & $<0.001$ & 0.14 & 2.8 & 1,92 & 0.097 & 0.04 \\
\hline A-Teasing $\rightarrow$ ARS $\rightarrow$ DC $\rightarrow$ Anxiety & 51.8 & 3,403 & $<0.001$ & 0.31 & 1.3 & 3,90 & 0.283 & 0.05 \\
\hline A-Teasing $\rightarrow$ Self-Esteem & 32.4 & 1, 405 & $<0.001$ & 0.09 & 2.3 & 1,92 & 0.133 & 0.05 \\
\hline A-Teasing $\rightarrow$ ARS $\rightarrow$ DC $\rightarrow$ Self-Esteem & 58.2 & 3,403 & $<0.001$ & 0.35 & 1.7 & 3,90 & 0.179 & 0.08 \\
\hline
\end{tabular}

A-Teasing, appearance teasing frequency (perceptions of teasing scale); ARS, appearance-based rejection sensitivity; DC, dysmorphic concerns. 
For the prediction of current degrees of depressive symptoms in the female subsample, early appearance teasing frequency was a significant and positive predictor $(c=2.48, p<0.001)$ in the total effect model without consideration of the mediators. However, the explained variance increased by $\Delta R^{2}=0.20$ when the mediators, dysmorphic concerns and ARS, were included in the model. All three possible indirect effects were significant [POTS-TF $\rightarrow$ ARS-D $\rightarrow$ PHQ-9: $b=0.58,95 \%$ CI $(0.31 ; 0.92)$; POTS-TF $\rightarrow$ ARS-D $\rightarrow$ DCQ $\rightarrow$ PHQ-9: $b=0.32,95 \%$ CI (0.20; $0.50)$; POTS-TF $\rightarrow$ DCQ $\rightarrow$ PHQ-9: $b=0.58,95 \%$ CI $(0.37$; $0.86)]$. Correspondingly, the total indirect effect was significant $[b=1.48,95 \%$ CI $(1.09 ; 1.92)]$, whereas the direct effect was reduced by inclusion of the mediators but remained significant, too $\left[c^{\prime}=1.00, p=0.002 ; 95 \% \mathrm{CI}(0.38 ; 1.62)\right]$.

For the degree of anxiety symptoms in women, the pattern was comparable: Early appearances teasing experiences significantly predicted higher current anxiety $(c=2.01, p<0.001)$ in the total effect model. Inclusion of the appearance-related mediators increased the explained variance by $\Delta R^{2}=0.17$. Again, all three indirect effects were significant [POTS-TF $\rightarrow$ ARS-D $\rightarrow$ GAD-7: $b=0.35,95 \%$ CI $(0.15 ; 0.61)$; POTS-TF $\rightarrow$ ARS-D $\rightarrow$ DCQ $\rightarrow$ GAD-7: $b=0.29,95 \%$ CI $(0.18 ; 0.44) ;$ POTS-TF $\rightarrow$ DCQ $\rightarrow$ GAD-7: $b=0.53,95 \%$ CI $(0.34 ; 0.79)]$. The total indirect effect $[b=1.18,95 \%$ CI $(0.87 ; 1.54)]$ was stronger than the total direct effect $\left[c^{\prime}=0.84, p=0.003 ; 95 \%\right.$ CI $\left.(0.29 ; 1.38)\right]$. However, the direct effect remained significant.

With regard to self-esteem, we observed a slightly different pattern. While the total effect model showed that early appearance teasing experiences had a significant negative impact $(c=-2.08, p<0.001)$ on the female participants' self-esteem, indirect effects of the mediators were even stronger for this mental health outcome. When including the mediators, the amount of explained variance increased by $\Delta R^{2}=0.26$. Again, each of the three indirect effects was significant [POTS-TF $\rightarrow$ ARS-D $\rightarrow$ RSES: $b=-1.14$, 95\% CI $(-1.60 ;-0.77)$; POTS-TF $\rightarrow$ ARS-D $\rightarrow$ DCQ $\rightarrow$ RSES: $b=-0.24$, 95\% CI $(-0.40$; $-0.13)$; POTS-TF $\rightarrow$ DCQ $\rightarrow$ RSES: $b=-0.44,95 \%$ CI $(-0.72$; $-0.24)]$. In line with this, the total indirect effect was significant, $b=-1.83 ; 95 \%$ CI $[-2.39 ;-1.36]$. However, as a result of the included mediators, the direct effect of appearance teasing on self-esteem was no longer significant [ $c^{\prime}=-0.25, p=0.473 ; 95 \%$ CI $(-0.93 ; 0.43)]$.

For the male subsample, the results differed from those of the female group. Early appearance teasing increased ARS $\left(a_{1}=2.01\right.$, $p=0.003)$. Besides, ARS $\left(d_{12}=0.25, p=0.006\right)$, but not appearance teasing $\left(a_{2}=1.07, p=0.061\right)$, predicted dysmorphic concerns. However, there were neither significant direct effects of early appearance teasing experiences on current mental health outcomes, nor any significant indirect effects via the mediators. ARS-D and DCQ values predicted none of the mental health outcomes in the serial mediation models ( $p s>0.167$ ).

The total effect models were not significant for depression, anxiety, and self-esteem in the male subgroup. Here, the amount of increased explained variance never surpassed $\Delta R^{2}=0.03$ when the appearance-related mediators were included in the regression model (see Table 4). Further, none of the indirect effects via any of the mediators was significant [total indirect effects: $Y=$ PHQ-9: $b=0.19,95 \%$ CI $(-0.29 ; 0.88) ; Y=$ GAD-7: $b=0.19,95 \% \mathrm{CI}$ $(-0.30 ; 0.88) ; Y=$ RSES: $b=-0.45,95 \%$ CI $(-1.12 ; 0.20)]$.

\section{Control Analyses}

We re-conducted all serial mediation analyses while controlling for possible influences of BMI, age, and relationship-status (binary code: 0 = no relationship; 1 = relationship) as covariates. The pattern of results remained stable in those analyses. There were no changes in the significance levels or effects of any of the aforementioned models, predictors, or mediators.

\section{DISCUSSION}

In the present study, we examined gender differences in appearance teasing, its relationship with two body image variables (ARS and dysmorphic concerns), and associations with different mental health outcomes - depression, anxiety, and self-esteem. Against the background of previous etiological models for body image disorders (e.g., Neziroglu et al., 2008) and recent empirical findings (e.g., Lavell et al., 2014; Webb et al., 2015; Densham et al., 2017), we tested potential serial mediation effects of ARS and dysmorphic concerns in the relationship of appearance teasing and mental health. A focus of these investigations was an analysis of potential genderspecific differences.

Overall, the ratio of men and women reporting any teasing events did not differ significantly. This finding contradicts previous reports on higher prevalence of appearance teasing in girls and women compared to boys and men (Farrow and Fox, 2011; Almenara and Ježek, 2015). However, in line with results from previous research, women showed higher impairments in body image variables (e.g., Feingold and Mazzella, 1998; Demarest and Allen, 2000; Buhlmann et al., 2010), as well as stronger mental health impairments and lower self-esteem compared to men (Piccinelli and Wilkinson, 2000; McMullin and Cairney, 2004; Bleidorn et al., 2016; von Soest et al., 2016).

Regarding the bivariate relationships of early teasing experiences with body image variables, we observed that appearance teasing frequency was significantly associated with higher degrees of adult ARS and stronger dysmorphic concerns in both, men and women. These findings support assumptions about the long lasting impact of early victimization experiences, especially with regard to body image (Eisenberg et al., 2003; Menzel et al., 2010; Liang et al., 2011; Webb et al., 2015). For the relationship between appearance teasing and mental health, effects were less pronounced in men compared to women. Thus, men seem to experience fewer mental health impairments because of early appearance teasing, confirming earlier findings (e.g., Gruber and Fineran, 2008; Carbone-Lopez et al., 2010; Feragen and Stock, 2016). In addition, the associations of body image variables and mental health were strong in women, but weak and not significant in men. Again, these findings are in line with previous research (Furnham et al., 2002; McMullin and Cairney, 2004).

In the following exploration of the serial mediation model, we observed additional effects that contribute to the knowledge 
about potential pathways in the relationship of appearance teasing on mental health: One observation concerns a gender-invariant mediation effect in the first part of the model (POTS $\rightarrow$ ARS $\rightarrow$ DCQ). For both, men and women, ARS played a crucial role in predicting dysmorphic concerns elicited by appearance teasing. These results confirm previous findings regarding the important role of the dispositional trait of ARS as a mediator in the development of dysmorphic concerns and BDD symptoms (Park et al., 2010; Lavell et al., 2014; Webb et al., 2015; Densham et al., 2017). Thus, the findings highlight the important role of ARS in the initiation and perpetuation of body image disorders. The construct could therefore be a factor to explore for potential inclusion in advanced etiological models of body image disorders - independent of gender.

However, the present study suggests that there are in fact gender differences regarding the other pathways of the serial mediation model: In women, appearance teasing experiences were significantly related to current depression, anxiety, and low self-esteem. This relationship is further explained by mediation effects of ARS and dysmorphic concerns. Thus, early appearance teasing increases ARS with its enhanced fear of interpersonal rejection due to the appearance. ARS then fosters enhanced dysmorphic concerns (i.e., excessive worries about the own appearance). These worries then increase the likelihood of depressive and anxious feelings as well as lower self-esteem in adulthood. Especially for self-esteem in adult women, early appearance teasing no longer served as a predictor when the two body image mediators were included in the analysis. This serial mediation effect explains how ARS and dysmorphic concerns are phenomena that contribute to mental health issues in women. It further shows that appearance and body image still strongly contribute to self-esteem in women (Furnham et al., 2002; Calogero et al., 2007; Swami et al., 2010). This effect may be caused by the stronger internalization of beauty-ideals for female individuals (e.g., Vandenbosch and Eggermont, 2012) and stronger external exposure to exaggerated beauty standards (Grabe et al., 2008). Also, women have been shown to more readily remember appearance teasing than competency teasing (Agliata et al., 2007). This cognitive accessibility could constitute another vulnerability factor for the continued negative effects of early appearance teasing experiences on the mental health of adult females.

In the male subsample, the overall pattern was different: Despite of negative effects on ARS and dysmorphic concerns, early appearance teasing was not significantly related to any of the mental health variables. In addition, the body image variables were not associated with mental health outcomes in men. This shows, that although appearance teasing has specific negative effects on body image, these effects do not generalize to general mental health outcomes in men, constituting a substantial gender difference.

Several explanations can be consulted for this gender difference: male mental health might generally be comprised of more sources of self-esteem, such as academic, financial, or athletic performances (Bolognini et al., 1996), limiting the impact of body image and of appearance teasing on mental health. Thus, it would be interesting to examine, whether teasing in other domains (e.g., competency) has stronger effects on adult mental health in men.

Another explanation may lie in predominant tendencies for externalizing behaviors rather than internalizing concerns in response to victimization (Llewellyn and Rudolph, 2014; Gregg et al., 2016; Stubbs-Richardson et al., 2018). Boys may be more prone to cope with victimization by showing dysfunctional behaviors (e.g., substance use, aggressive behaviors), or perpetuate victimization to third persons. In contrast, girls show more avoidance behaviors (Llewellyn and Rudolph, 2014), practice self-blame, and self-harming eating behaviors (Haines et al., 2006; Ata et al., 2007).

In addition, gender differences in coping abilities could contribute to the observed effects (Tamres et al., 2002; Boulton, 2013). For example, women tend to use more rumination, a dysfunctional emotion regulation strategy that can explain gender differences in mental health (e.g., Aldao et al., 2010; Opwis et al., 2017). Women also tend to seek social support as a strategy to cope with emotional and social stressors (Tamres et al., 2002). Therefore, losing this resource due to elevated ARS specifically infringes available coping strategies that might help precluding mental health problems in women. The identification of these possible resilience and vulnerability factors that moderate gender-specific appearance teasing effects should be subjects of future studies.

With regard to therapeutic implications, the present findings highlight the need to address ARS in body image treatments in both genders, for example via behavioral experiments and cognitive restructuring. Especially for women, therapeutic work with ARS could indirectly improve dysmorphic concerns and associated general mental health. Behavioral experiments may be useful therapeutic tools to generate disconfirming evidence regarding fear of interpersonal rejection. In addition, the results hint at the importance of more prevention measures to avoid long term negative outcomes of appearance teasing in women. Interventions that establish more sources of self-esteem beyond appearance at an early age might enhance resilience in women. In addition, cognitive reframing of teasing experiences could help preventing mental health impairments in female victims of appearance teasing.

Despite of these promising results, the study is subject to several limitations. For example, the data in this study is cross-sectional and bases on a convenience sample with a larger proportion of female compared to male respondents. The known restrictions of online surveys apply to this study (e.g., a lack of control over the circumstances while filling in the questionnaire and possible biases regarding the sample; see Barratt et al., 2015). We controlled for possible confounding factors, such as extreme response latencies, with conservative data screening procedures. Still, we cannot rule out any probabilities of sampling biases, especially with regard to the few inclusion and exclusion criteria applied. For example, because of the announcement of the study as research on appearance, body image, and mental health, it is possible that there was a selection bias. The sample may be characterized by a special interest in these topics. Future studies should therefore particularly ensure the recruitment of representative samples. Further, we were not able to control for 
possible confounding effects of prior or current interventions (e.g., medication or psychotherapy) on the mental health status of the respondents.

Limitations also result from the retrospective reports of teasing. We cannot rule out the possibility of distorted reports of teasing due to the long latencies and possible memory biases, especially in older participants. Despite of our control analysis for age, this limitation has to be taken into account when appraising the findings of this study. Future studies on this topic should also aim at recruiting equal sample sizes for the male and female subgroups to ensure equal statistical power to detect significant effects in men and women. In general, this cross-sectional study constitutes a first step in understanding the gender-specific relationship of appearance teasing, body image variables, and mental health. Actual experimental and longitudinal research (e.g., with applications of ecological momentary assessment) should to be aspired to allow for veritable analyses of mechanistic processes in this research field.

Further, we did not assess sexual orientation. Therefore, we cannot provide answers about possible differences regarding the effects of appearance teasing on body image and mental health outcomes with respect to hetero-, homo-, bi-, or transsexual individuals. Earlier research has shown more victimizations and an increased importance of body image in sexual minorities, such as homosexual men (e.g., Gruber and Fineran, 2008; Frederick and Essayli, 2016). Therefore, future studies should take into account the possible moderating effects of sexual orientation in explanatory models.

However, the present study also has several strengths: It directly compares gender differences of appearance teasing and its effects on adult mental health. Earlier studies often focused on effects in adolescence and/or examined effects in female samples only. In addition, to our best knowledge, this was the first study to examine more complex mediation pathways regarding the role of two relevant body image variables, ARS as a dispositional factor and dysmorphic concerns as a cognitive factor, in explaining gender-specific effects of appearance teasing on various mental health outcomes. In addition, we tested all mediation models again, while controlling for potential influences of age, BMI, and relationship-status and obtained the same pattern of results. Thus, the effects have proven stable against possible influences of those common confounders with respect to body image, as well as mental health variables.

\section{CONCLUSION}

The present study observed gender-specific effects of early appearance teasing experiences on mental health in adulthood,

\section{REFERENCES}

Agliata, A. K., Tantleff-Dunn, S., and Renk, K. (2007). Interpretation of teasing during early adolescence. J. Clin. Psychol. 63, 23-30. doi: 10.1002/jclp. 20302

Aldao, A., Nolen-Hoeksema, S., and Schweizer, S. (2010). Emotion-regulation strategies across psychopathology: a meta-analytic review. Clin. Psychol. Rev. 30, 217-237. doi: 10.1016/j.cpr.2009.11.004 although men and women reported similar frequencies of teasing. While appearance teasing experiences have specific negative effects on the body image of both, women and men, the negative effects do not stretch out to more global mental health concerns in men. However, in women, ARS and dysmorphic concerns play an important mediating role in explaining negative effects of appearance teasing in childhood and adolescence on mental health in adulthood. The study highlights the role of ARS as an important construct in the etiology of body image disorders that should therefore be a specific target of therapeutic interventions for this array of mental disorders. However, future research needs to explore moderators that serve as resilience and vulnerability factors regarding gender-specific negative effects of appearance teasing on mental health.

\section{DATA AVAILABILITY}

The raw data supporting the conclusions of this manuscript will be made available by the authors, without undue reservation, to any qualified researcher.

\section{ETHICS STATEMENT}

This study was carried out in accordance with the recommendations of "Richtlinien der Ethik-Kommission der Bergischen Universität Wuppertal." All subjects gave written informed consent in accordance with the Declaration of Helsinki. The protocol was approved by the Ethik-Kommission der Bergischen Universität Wuppertal (Reference No. MS/JE 180312 Schmidt 2).

\section{AUTHOR CONTRIBUTIONS}

JS drafted the manuscript with important contributions from AM, designed the study in collaboration with AM, and conducted the analyses. Both authors have approved the final manuscript to be published.

\section{ACKNOWLEDGMENTS}

The authors would like to thank Anna Schumacher and Jonathan Röpke for their assistance in the data collection and research process.

Almenara, C. A., and Ježek, S. (2015). The source and impact of appearance teasing: an examination by sex and weight status among early adolescents from the Czech Republic. J. Sch. Health 85, 163-170. doi: 10.1111/josh.12236

American Psychiatric Association (2013). Diagnostic and Statistical Manual of Mental Disorders (DSM-5). Arlington, VA: American Psychiatric Publishing. doi: 10.1176/appi.books.9780890425596

Ata, R. N., Ludden, A. B., and Lally, M. M. (2007). The effects of gender and family, friend, and media influences on eating behaviors and body image 
during adolescence. J. Youth Adolesc. 36, 1024-1037. doi: 10.1007/s10964-0069159-x

Barratt, M. J., Ferris, J. A., and Lenton, S. (2015). Hidden populations, online purposive sampling, and external validity: taking off the blindfold. Field Methods 27, 3-21. doi: 10.1177/1525822X14526838

Baudson, T. G., Weber, K. E., and Freund, P. A. (2016). More than only skin deep: appearance self-concept predicts most of secondary school students' self-esteem. Front. Psychol. 7:1568. doi: 10.3389/fpsyg.2016.01568

Biby, E. L. (1998). The relationship between body dysmorphic disorder and depression, self-esteem, somatization, and obsessive-compulsive disorder. J. Clin. Psychol. 54, 489-499. doi: 10.1002/(SICI)1097-4679(199806)54:4<489:: AID-JCLP10>3.0.CO;2-B

Bleidorn, W., Arslan, R. C., Denissen, J. J., Rentfrow, P. J., Gebauer, J. E., Potter, J., et al. (2016). Age and gender differences in self-esteem-A cross-cultural window. J. Pers. Soc. Psychol. 111, 396-410. doi: 10.1037/pspp0000078

Bohne, A., Keuthen, N. J., Wilhelm, S., Deckersbach, T., and Jenike, M. A. (2002). Prevalence of symptoms of body dysmorphic disorder and its correlates: a crosscultural comparison. Psychosomatics 43, 486-490. doi: 10.1176/appi.psy.43. 6.486

Bolognini, M., Plancherel, B., Bettschart, W., and Halfon, O. (1996). Self-esteem and mental health in early adolescence: development and gender differences. J. Adolesc. 19, 233-245. doi: 10.1006/jado.1996.0022

Boulton, M. J. (2013). Associations between adults' recalled childhood bullying victimization, current social anxiety, coping, and self-blame: evidence for moderation and indirect effects. Anxiety Stress Coping 26, 270-292. doi: 10. 1080/10615806.2012.662499

Bowker, J. C., Thomas, K. K., Spencer, S. V., and Park, L. E. (2013). Examining appearance-based rejection sensitivity during early adolescence. J. Res. Adolesc. 23, 375-388. doi: 10.1111/jora. 12003

Buhlmann, U., Cook, L. M., Fama, J. M., and Wilhelm, S. (2007). Perceived teasing experiences in body dysmorphic disorder. Body Image 4, 381-385. doi: 10.1016/ j.bodyim.2007.06.004

Buhlmann, U., Glaesmer, H., Mewes, R., Fama, J. M., Wilhelm, S., Brähler, E., et al. (2010). Updates on the prevalence of body dysmorphic disorder: a populationbased survey. Psychiatry Res. 178, 171-175. doi: 10.1016/j.psychres.2009. 05.002

Buhlmann, U., Wilhelm, S., Glaesmer, H., Mewes, R., Brähler, E., and Rief, W. (2011). Perceived appearance-related teasing in body dysmorphic disorder: a population-based survey. Int. J. Cogn. Ther. 4, 342-348. doi: 10.1521/ijct.2011. 4.4.342

Calogero, R. M., Boroughs, M., and Thompson, J. K. (2007). "The impact of Western beauty ideals on the lives of women: a sociocultural perspective," in The Body Beautiful, eds V. Swami and A. Furnham (London: Palgrave Macmillan), 259-298.

Calogero, R. M., Park, L. E., Rahemtulla, Z. K., and Williams, K. C. (2010). Predicting excessive body image concerns among British university students: the unique role of appearance-based rejection sensitivity. Body Image 7, 78-81. doi: 10.1016/j.bodyim.2009.09.005

Carbone-Lopez, K., Esbensen, F. A., and Brick, B. T. (2010). Correlates and consequences of peer victimization: gender differences in direct and indirect forms of bullying. Youth Violence Juv. Justice 8, 332-350. doi: 10.1177/ 1541204010362954

Cash, T. F. (1995). Developmental teasing about physical appearance: retrospective descriptions and relationships with body image. Soc. Behav. Pers. 23, 123-130. doi: $10.2224 /$ sbp.1995.23.2.123

Cohen, J. (1988). Statistical Power Analysis for the Behavioral Sciences. Hillsdale, NJ: L. Lawrence Earlbaum Associates.

Demarest, J., and Allen, R. (2000). Body image: gender, ethnic, and age differences. J. Soc. Psychol. 140, 465-472. doi: 10.1080/00224540009600485

Densham, K., Webb, H. J., Zimmer-Gembeck, M. J., Nesdale, D., and Downey, G. (2017). Early adolescents' body dysmorphic symptoms as compensatory responses to parental appearance messages and appearance-based rejection sensitivity. Body Image 23, 162-170. doi: 10.1016/j.bodyim.2017. 09.005

Eisenberg, M. E., Neumark-Sztainer, D., and Story, M. (2003). Associations of weight-based teasing and emotional well-being among adolescents. Arch. Pediatr. Adolesc. Med. 157, 733-738. doi: 10.1001/archpedi.157. 8.733
Faith, M. A., Storch, E. A., Roberti, J. W., and Ledley, D. R. (2008). Recalled childhood teasing among non-clinical, non-college adults. J. Psychopathol. Behav. Assess. 30, 171-179. doi: 10.1007/s10862-007-9062-0

Farrow, C. V., and Fox, C. L. (2011). Gender differences in the relationships between bullying at school and unhealthy eating and shape-related attitudes and behaviours. Br. J. Educ. Psychol. 81, 409-420. doi: 10.1348/000709910X525804

Faul, F., Erdfelder, E., Lang, A. G., and Buchner, A. (2007). G* Power 3: a flexible statistical power analysis program for the social, behavioral, and biomedical sciences. Behav. Res. Methods 39, 175-191. doi: 10.3758/BF0319 3146

Feingold, A., and Mazzella, R. (1998). Gender differences in body image are increasing. Psychol. Sci. 9, 190-195. doi: 10.1111/1467-9280.00036

Feragen, K. B., and Stock, N. M. (2016). A longitudinal study of 340 young people with or without a visible difference: the impact of teasing on self-perceptions of appearance and depressive symptoms. Body Image 16, 133-142. doi: 10.1016/j. bodyim.2016.01.003

Ford, R., King, T., Priest, N., and Kavanagh, A. (2017). Bullying and mental health and suicidal behaviour among 14-to 15-year-olds in a representative sample of Australian children. Aust. N. Z. J. Psychiatry 51, 897-908. doi: 10.1177/ 0004867417700275

Frederick, D. A., and Essayli, J. H. (2016). Male body image: the roles of sexual orientation and body mass index across five national US studies. Psychol. Men Masc. 17, 336-351. doi: 10.1037/men0000031

Furnham, A., Badmin, N., and Sneade, I. (2002). Body image dissatisfaction: gender differences in eating attitudes, self-esteem, and reasons for exercise. J. Psychol. 136, 581-596. doi: 10.1080/00223980209604820

Gentile, B., Grabe, S., Dolan-Pascoe, B., Twenge, J. M., Wells, B. E., and Maitino, A. (2009). Gender differences in domain-specific self-esteem: a meta-analysis. Rev. Gen. Psychol. 13, 34-45. doi: 10.1037/a0013689

Gini, G., and Pozzoli, T. (2009). Association between bullying and psychosomatic problems: a meta-analysis. Pediatrics 123, 1059-1065. doi: 10.1542/peds.20081215

Gleason, J. H., Alexander, A. M., and Somers, C. L. (2000). Later adolescents' reaction to three types of childhood teasing: relation with self-esteem and body image. Soc. Behav. Pers. 28, 471-479. doi: 10.2224/sbp.2000.28.5.471

Grabe, S., Ward, L. M., and Hyde, J. S. (2008). The role of the media in body image concerns among women: a meta-analysis of experimental and correlational studies. Psychol. Bull. 134, 460-476. doi: 10.1037/0033-2909. 134.3.460

Gräfe, K., Zipfel, S., Herzog, W., and Löwe, B. (2004). Screening psychischer Störungen mit dem "Gesundheitsfragebogen für Patienten (PHQ-D)“. Diagnostica 50, 171-181. doi: 10.1026/0012-1924.50.4.171

Greenleaf, C., Petrie, T. A., and Martin, S. B. (2014). Relationship of weight-based teasing and adolescents' psychological well-being and physical health. J. Sch. Health 84, 49-55. doi: 10.1111/josh.12118

Gregg, D. H., Somers, C. L., Pernice-Duca, F., and Van Dale, K. G. (2016). Teasing experiences and risk-taking: gender and self-esteem as moderator and mediator. J. Sch. Violence 15, 365-385. doi: 10.1080/15388220.2015.1054935

Gruber, J. E., and Fineran, S. (2008). Comparing the impact of bullying and sexual harassment victimization on the mental and physical health of adolescents. Sex Roles 59, 1-13. doi: 10.1007/s11199-008-9431-5

Haines, J., Neumark-Sztainer, D., Eisenberg, M. E., and Hannan, P. J. (2006). Weight teasing and disordered eating behaviors in adolescents: longitudinal findings from Project EAT (Eating Among Teens). Pediatrics 117:e209-15. doi: $10.1542 /$ peds.2005-1242

Hawker, D. S., and Boulton, M. J. (2000). Twenty years' research on peer victimization and psychosocial maladjustment: a meta-analytic review of crosssectional studies. J. Child Psychol. Psychiatry 41, 441-455. doi: 10.1111/14697610.00629

Hayden-Wade, H. A., Stein, R. I., Ghaderi, A., Saelens, B. E., Zabinski, M. F., and Wilfley, D. E. (2005). Prevalence, characteristics, and correlates of teasing experiences among overweight children vs. non-overweight peers. Obes. Res. 13, $1381-1392$.

Hayes, A. F. (2013). Introduction to Mediation, Moderation, and Conditional Process Analysis: A Regression-Based Approach. New York: Guilford Publications.

Hyde, J. S., Mezulis, A. H., and Abramson, L. Y. (2008). The ABCs of depression: integrating affective, biological, and cognitive models to explain the emergence 
of the gender difference in depression. Psychol. Rev. 115, 291-313. doi: 10.1037/ 0033-295X.115.2.291

Jackson, T. D., Grilo, C. M., and Masheb, R. M. (2000). Teasing history, onset of obesity, current eating disorder psychopathology, body dissatisfaction, and psychological functioning in binge eating disorder. Obes. Res. 8, 451-458. doi: 10.1038/oby.2000.56

Jensen, C. D., and Steele, R. G. (2010). Validation of the perceptions of teasing scale (POTS) in a preadolescent sample: associations with attitudes toward physical activity. Child. Health Care 39, 249-265. doi: 10.1080/02739615.2010. 515925

Jones, D. C., and Crawford, J. K. (2006). The peer appearance culture during adolescence: gender and body mass variations. J. Youth Adolesc. 35:243. doi: 10.1007/s10964-005-9006-5

Jones, D. C., Newman, J. B., and Bautista, S. (2005). A three-factor model of teasing: the influence of friendship, gender, and topic on expected emotional reactions to teasing during early adolescence. Soc. Dev. 14, 421-439. doi: 10.1111/j.14679507.2005.00309.x

Keery, H., Boutelle, K., van den Berg, P., and Thompson, J. K. (2005). The impact of appearance-related teasing by family members. J. Adolesc. Health 37, 120-127. doi: 10.1016/j.jadohealth.2004.08.015

Kelly, M. M., Didie, E. R., and Phillips, K. A. (2014). Personal and appearance-based rejection sensitivity in body dysmorphic disorder. Body Image 11, 260-265. doi: 10.1016/j.bodyim.2014.03.004

Keltner, D., Capps, L., Kring, A. M., Young, R. C., and Heerey, E. A. (2001). Just teasing: a conceptual analysis and empirical review. Psychol. Bull. 127, 229-248. doi: 10.1037/0033-2909.127.2.229

Kessler, R. C., Birnbaum, H. G., Shahly, V., Bromet, E., Hwang, I., McLaughlin, K. A., et al. (2010). Age differences in the prevalence and co-morbidity of DSM-IV major depressive episodes: results from the WHO world mental health survey initiative. Depress. Anxiety 27, 351-364. doi: 10.1002/da. 20634

Kochenderfer, B. J., and Ladd, G. W. (1996). Peer victimization: manifestations and relations to school adjustment in kindergarten. J. Sch. Psychol. 34, 267-283. doi: $10.1017 /$ S0954579410000283

Lavell, C. H., Zimmer-Gembeck, M. J., Farrell, L. J., and Webb, H. (2014). Victimization, social anxiety, and body dysmorphic concerns: appearancebased rejection sensitivity as a mediator. Body Image 11, 391-395. doi: 10.1016/ j.bodyim.2014.06.008

Leiner, D. J. (2018). SoSci Survey (Program-Version 3.1.01-i) [Computer software]. Available at: https://www.soscisurvey.de

Liang, V. X., Jackson, A. C., and McKenzie, V. L. (2011). The effects of teasing in childhood or adolescence on young adults' body image. Educ. Dev. Psychol. 28, 101-115. doi: 10.1375/aedp.28.2.101

Little, R. J. (1988). A test of missing completely at random for multivariate data with missing values. J. Am. Stat. Assoc. 83, 1198-1202. doi: 10.1080/01621459. 1988.10478722

Llewellyn, N., and Rudolph, K. D. (2014). Individual and sex differences in the consequences of victimization: moderation by approach and avoidance motivation. Dev. Psychol. 50, 2210-2220. doi: 10.1037/a0037353

Lopez-Guimera, G., Levine, M. P., Sánchez-Carracedo, D., and Fauquet, J. (2010). Influence of mass media on body image and eating disordered attitudes and behaviors in females: a review of effects and processes. Media Psychol. 13, 387-416. doi: 10.1080/15213269.2010.525737

Losekam, S., Kraeling, S., Goetzky, B., Rief, W., and Hilbert, A. (2017). Evaluation of the German version of the Perception of Teasing Scale (POTS). Marburg: University of Margburg.

Löwe, B., Decker, O., Müller, S., Brähler, E., Schellberg, D., Herzog, W., et al. (2008). Validation and standardization of the generalized anxiety disorder screener (GAD-7) in the general population. Med. Care 56, 266-274. doi: 10.1097/MLR. 0b013e318160d093

Marcotte, D., Fortin, L., Potvin, P., and Papillon, M. (2002). Gender differences in depressive symptoms during adolescence: role of gender-typed characteristics, self-esteem, body image, stressful life events, and pubertal status. J. Emot. Behav. Disord. 10, 29-42. doi: 10.1177/106342660201000104

Martin, A., Rief, W., Klaiberg, A., and Braehler, E. (2006). Validity of the brief patient health questionnaire mood scale (PHQ-9) in the general population. Gen. Hosp. Psychiatry 28, 71-77. doi: 10.1016/j.genhosppsych.2005. 07.003
McCabe, R. E., Miller, J. L., Laugesen, N., Antony, M. M., and Young, L. (2010). The relationship between anxiety disorders in adults and recalled childhood teasing. J. Anxiety Disord. 24, 238-243. doi: 10.1016/j.janxdis.2009.11.002

McLaren, L., Beck, C. A., Patten, S. B., Fick, G. H., and Adair, C. E. (2008). The relationship between body mass index and mental health. Soc. Psychiatry Psychiatr. Epidemiol. 43, 63-71. doi: 10.1007/s00127-007-0269-x

McMullin, J. A., and Cairney, J. (2004). Self-esteem and the intersection of age, class, and gender. J. Aging Stud. 18, 75-90. doi: 10.1016/j.jaging.2003.09.006

Menzel, J. E., Schaefer, L. M., Burke, N. L., Mayhew, L. L., Brannick, M. T., and Thompson, J. K. (2010). Appearance-related teasing, body dissatisfaction, and disordered eating: a meta-analysis. Body Image 7, 261-270. doi: 10.1016/j. bodyim.2010.05.004

Neziroglu, F., Khemlani-Patel, S., and Veale, D. (2008). Social learning theory and cognitive behavioral models of body dysmorphic disorder. Body Image 5, 28-38. doi: 10.1016/j.bodyim.2008.01.002

Opwis, M., Schmidt, J., Martin, A., and Salewski, C. (2017). Gender differences in eating behavior and eating pathology: the mediating role of rumination. Appetite 110, 103-107. doi: 10.1016/j.appet.2016.12.020

Park, L. E. (2007). Appearance-based rejection sensitivity: implications for mental and physical health, affect, and motivation. Pers. Soc. Psychol. Bull. 33, 490-504. doi: $10.1177 / 0146167206296301$

Park, L. E., Calogero, R. M., Harwin, M. J., and DiRaddo, A. M. (2009). Predicting interest in cosmetic surgery: interactive effects of appearance-based rejection sensitivity and negative appearance comments. Body Image 6, 186-193. doi: 10.1016/j.bodyim.2009.02.003

Park, L. E., Calogero, R. M., Young, A. F., and DiRaddo, A. M. (2010). Appearancebased rejection sensitivity predicts body dysmorphic disorder symptoms and cosmetic surgery acceptance. J. Soc. Clin. Psychol. 29, 489-509. doi: 10.1521/ jscp.2010.29.5.489

Phares, V., Steinberg, A. R., and Thompson, J. K. (2004). Gender differences in peer and parental influences: body image disturbance, self-worth, and psychological functioning in preadolescent children. J. Youth Adolesc. 33, 421-429. doi: 10. 1023/B:JOYO.0000037634.18749.20

Phillips, K. A. (2005). The Broken Mirror: Understanding and Treating Body Dysmorphic Disorder. New York, NY: Oxford University Press.

Piccinelli, M., and Wilkinson, G. (2000). Gender differences in depression: critical review. Br. J. Psychiatry 177, 486-492. doi: 10.1192/bjp.177.6.486

Schieber, K., Kollei, I., de Zwaan, M., and Martin, A. (2018). The dysmorphic concern questionnaire in the german general population: psychometric properties and normative data. Aesthet. Plast. Surg. 42, 1412-1420. doi: 10.1007/ s00266-018-1183-1

Schmidt, J., and Martin, A. (2017). Du magst mich nicht, weil ich hässlich bin! Aussehensbezogene Zurückweisungssensitivität und Validierung der deutschsprachigen Appearance-based Rejection Sensitivity Scale (ARS-D). Zeitschrift für Klinische Psychologie und Psychotherapie 46, 157-168. doi: 10. 1026/1616-3443/a000433

Schwartz, D. J., Phares, V., Tantleff-Dunn, S., and Thompson, J. K. (1999). Body image, psychological functioning, and parental feedback regarding physical appearance. Int. J. Eat. Disord. 25, 339-343. doi: 10.1002/(SICI)1098108X(199904)25:3<339::AID-EAT13>3.0.CO;2-V

Sinclair, S. J., Blais, M. A., Gansler, D. A., Sandberg, E., Bistis, K., and LoCicero, A. (2010). Psychometric properties of the rosenberg self-esteem scale: overall and across demographic groups living within the United States. Eval. Health Prof. 33, 56-80. doi: 10.1177/0163278709356187

Stangier, U., Janich, C., Adam-Schwebe, S., Berger, P., and Wolter, M. (2003). Screening for body dysmorphic disorder in dermatological outpatients. Dermatol. Psychosom. 4, 66-71. doi: 10.1159/000072194

Storch, E. A., Brassard, M. R., and Masia-Warner, C. L. (2003). The relationship of peer victimization to social anxiety and loneliness in adolescence. Child Study J. $33,1-18$.

Stubbs-Richardson, M., Sinclair, H. C., Goldberg, R. M., Ellithorpe, C. N., and Amadi, S. C. (2018). Reaching out versus lashing out: examining gender differences in experiences with and responses to bullying in high school. Am. J. Crim. Justice 43, 39-66. doi: 10.1007/s12103-0179408-4

Swami, V., Frederick, D. A., Aavik, T., Alcalay, L., Allik, J., Anderson, D., et al. (2010). The attractive female body weight and female body dissatisfaction in 26 countries across 10 world regions: results of the international body 
project I. Pers. Soc. Psychol. Bull. 36, 309-325. doi: 10.1177/014616720935 9702

Tamres, L. K., Janicki, D., and Helgeson, V. S. (2002). Sex differences in coping behavior: a meta-analytic review and an examination of relative coping. Pers. Soc. Psychol. Rev. 6, 2-30. doi: 10.1207/S15327957PSPR0601_1

Thompson, I., Hong, J. S., Lee, J. M., Prys, N. A., Morgan, J. T., and Udo-Inyang, I. (2018). A review of the empirical research on weight-based bullying and peer victimisation published between 2006 and 2016. Educ. Rev. 1-23. doi: 10.1080/ 00131911.2018.1483894

Thompson, J. K., Cattarin, J., Fowler, B., and Fisher, E. (1995). The perception of teasing scale (POTS): a revision and extension of the physical appearance related teasing scale (PARTS). J. Pers. Assess. 65, 146-157. doi: 10.1207/ s15327752jpa6501_11

Vandenbosch, L., and Eggermont, S. (2012). Understanding sexual objectification: a comprehensive approach toward media exposure and girls' internalization of beauty ideals, self-objectification, and body surveillance. J. Commun. 62, 869-887. doi: 10.1111/j.1460-2466.2012.01667.x

Veale, D. (2004). Advances in a cognitive behavioural model of body dysmorphic disorder. Body Image 1, 113-125. doi: 10.1016/S1740-1445(03) 00009-3

Vogt Yuan, A. S. (2010). Body perceptions, weight control behavior, and changes in adolescents' psychological well-being over time: a longitudinal examination of gender. J. Youth Adolesc. 39, 927-939. doi: 10.1007/s10964-0099428-6

von Collani, G., and Herzberg, P. Y. (2003). Eine revidierte Fassung der deutschsprachigen Skala zum Selbstwertgefühl von Rosenberg. Zeitschrift für Differentielle und Diagnostische Psychologie 24, 3-7. doi: 10.1024//0170-1789. 24.1.3

von Soest, T., Wichstrøm, L., and Kvalem, I. L. (2016). The development of global and domain-specific self-esteem from age 13 to 31. J. Pers. Soc. Psychol. 110, 592-608. doi: 10.1037/pspp0000060

Webb, H. J., Zimmer-Gembeck, M. J., Mastro, S., Farrell, L. J., Waters, A. M., and Lavell, C. H. (2015). Young adolescents' body dysmorphic symptoms: associations with same-and cross-sex peer teasing via appearance-based rejection sensitivity. J. Abnorm. Child Psychol. 43, 1161-1173. doi: 10.1007/ s10802-014-9971-9

Webb, H. J., Zimmer-Gembeck, M. J., Waters, A. M., Farrell, L. J., Nesdale, D., and Downey, G. (2017). Pretty pressure" from peers, parents, and the media: a longitudinal study of appearance-based rejection sensitivity. J. Res. Adolesc. 27, 718-735. doi: 10.1111/jora.12310

Weingarden, H., Curley, E. E., Renshaw, K. D., and Wilhelm, S. (2017). Patientidentified events implicated in the development of body dysmorphic disorder. Body Image 21, 19-25. doi: 10.1016/j.bodyim.2017.02.003

Weingarden, H., and Renshaw, K. D. (2016). Body dysmorphic symptoms, functional impairment, and depression: the role of appearance-based teasing. J. Psychol. 150, 119-131. doi: 10.1080/00223980.2015.101 2144

Willitts, M., Benzeval, M., and Stansfeld, S. (2004). Partnership history and mental health over time. J. Epidemiol. Commun. Health 58, 53-58. doi: 10.1136/jech.58. 1.53

Zimmer-Gembeck, M. J., Webb, H. J., Farrell, L. J., and Waters, A. M. (2018). Girls' and boys' trajectories of appearance anxiety from age 10 to 15 years are associated with earlier maturation and appearancerelated teasing. Dev. Psychopathol. 30, 337-350. doi: 10.1017/S095457941700 0657

Conflict of Interest Statement: The authors declare that the research was conducted in the absence of any commercial or financial relationships that could be construed as a potential conflict of interest.

Copyright (c) 2019 Schmidt and Martin. This is an open-access article distributed under the terms of the Creative Commons Attribution License (CC BY). The use, distribution or reproduction in other forums is permitted, provided the original author(s) and the copyright owner(s) are credited and that the original publication in this journal is cited, in accordance with accepted academic practice. No use, distribution or reproduction is permitted which does not comply with these terms. 